\title{
AGROTÓXICOS EM ÁGUA E ALIMENTOS: RISCO À SAÚDE HUMANA
}

PALMA, Danielly Cristina de Andrade. Docente do curso de Enfermagem da Universidade Federal de Mato Grosso do Sul/Câmpus Coxim. Av. Márcio de Lima Nantes, s/n - Vila da Barra, CEP 79400-000.

E-mail: danielly_palma@yahoo.com.br.

LOURENCETTI, Carolina. Docente do Instituto Federal de Educação, Ciência e Tecnologia de São Paulo/ Câmpus Avançado de Matão. Rua José Bonifácio, 1176 - Centro, CEP 15990-040. E-mail: carollourencetti@yahoo.com.br.

\section{Resumo}

Considerando o risco da exposição humana aos agrotóxicos pela ingestão de água e alimentos contaminados, este trabalho faz uma breve revisão na literatura sobre estudos desenvolvidos no Brasil que apresentam dados de agrotóxicos nos recursos hídricos e em amostras biológicas, além de casos de intoxicação humana. O Brasil é o maior consumidor de agrotóxicos entre os países em desenvolvimento, sendo que o seu intenso uso no processo de produção agropecuária, entre outras aplicações, pode resultar em diversos transtornos emodificações para o ambiente, refletindo na qualidade de vida da população. A problemática do uso intensivo de agrotóxico deveria ser debatida entre órgãos gestores competentes e sociedade para que medidas urgentes sejam tomadas e maiores danos, futuramente, sejam evitados.

Palavras-chave: Dispersão ambiental; Exposição humana; Programa de Análise de Resíduos de Agrotóxicos emAlimentos.

\begin{abstract}
Taking into account the risk of human exposition to pesticides by ingestion of contaminated water and food, this study presents a brief review about Brazilian studies regarding pesticides in water, in biological matrices, and in human intoxication cases. Brazil is the greatest consumer of pesticides among the developing countries, and the intensive use of these compounds in agriculture, among other applications, can result in changes tothe environment, reflecting in the population's life. The problem of pesticide intensive use should be discussed between governmental institutions and general population in order to consider urgent procedures avoiding future damages.
\end{abstract}

KEYWORDs: Environmental dispersion; Human exposure; Program of Analysis for Pesticide Residues in Food. 


\section{INTRODUÇÃo}

O uso de agrotóxicos tem sido a principal ferramenta no combate às "pragas" da agricultura, contribuindo assim para a manutenção das grandes lavouras de monoculturas espalhadas pelo mundo, obtenção de alta eficiência produtiva e retorno econômico. Os agrotóxicos são agentes poluidores e podem resultar em sérios problemas de saúde humana e ambiental, por isso este tema tem se tornado um dos principais focos de estudos da comunidade científica mundial (CALDAS e SOUZA, 2000; LAABS et al., 2002; GRUTZMACHER et al., 2008).

Após a aplicação dos agrotóxicos, somente uma pequena parte atinge a peste-alvo, enquanto o restante pode atingir outros compartimentos ambientais, como atmosfera, solo e recursos hídricos. A presença dos agrotóxicos nesses compartimentos depende de diversos mecanismos de retenção, transformação e transporte, juntamente com a forma de manejo, condições climáticas e propriedades do solo e dos agrotóxicos (SPADOTTO, 2006). Assim, diferentes princípios ativos apresentam diferentes comportamentos nos distintos compartimentos ambientais, podendo ou não persistir e ser disperso no ambiente. É importante ressaltar que, além do diferente comportamento no ambiente, os agrotóxicos também apresentam diferente toxicidade aos humanos, aos animais e aos organismos aquáticos.

Apesar da importância da agricultura para a economia, a ocupação do meio rural de forma desordenada e o emprego de tecnologias inadequadas podem provocar inúmeros impactos ao solo e aos recursos hídricos, podendo refletir na qualidade das águas superficiais e subterrâneas (GUNNINGHAM e SINCLAR, 2005; SANTOS NETO e SIQUEIRA, 2005).

A contaminação dos recursos hídricos pode provocar graves problemas à saúde pública, principalmente quando este recurso é utilizado para o abastecimento público. Aingestão diária de água e de alimentos contaminados por agrotóxicos pode provocar o acúmulo dessas substâncias no organismo humano, podendo causar graves doenças, como certos tipos de cânceres e distúrbios no sistema endócrino.

Considerando o risco da exposição humana aos agrotóxicos pela ingestão de água e alimentos contaminados, este trabalho faz uma breve revisão sobre estudos desenvolvidos no Brasil que apresentam dados de agrotóxicos nos recursos hídricos e em amostras biológicas, além de casos de intoxicação humana.

\section{INTENSIDADE DO USO DE AGROTÓXICOS}

Os agrotóxicos assumem caráter destacado como contaminantes pela intensidade de consumo no país (FERNANDES NETO e SARCINELLI, 2009). Os países em desenvolvimento representam $30 \%$ de todo o mercado global consumidor de agrotóxicos, sendo o Brasil o maior mercado consumidor individual dentre estes países, equivalente à metade de todo o consumo da America Latina (PERES et al., 2007).

O Brasil tem o registro de 1.500 produtos comerciais; destes, 673 estão no mercado. Entre esses diferentes produtos comerciais há a presença de agrotóxicos de diferentes classes químicas e modo de ação, apresentando também diferentes classificações toxicológicas e de risco ambiental. Em 2009, foram comercializadas 725 mil toneladas de produtos formulados. Considerando os ingredientes ativos, foram comercializadas 335.816 toneladas. As principais culturas consumidoras de agrotóxicos são as de soja (48\%), milho (11\%), cana-de-açúcar (8\%), algodão (7\%), café $(4 \%)$ e citros $(3 \%)$. O Estado de Mato Grosso é líder em vendas de agrotóxicos (20\%), seguido pelo Estado de São Paulo (15\%), Paraná (14\%), Rio Grande do Sul (11\%), Goiás (10\%) e Minas Gerais (9\%). O mercado de agrotóxicos no Brasil em 2009 movimentou US\$ 6,6 bilhões (MENTEN et al., 2010).

O consumo de agrotóxicos no Brasil tem crescido ano após ano, mas a área plantada não tem aumentado na mesma proporção. Isso significa que o consumo de agrotóxicos utilizado por hectare tem aumentado, agravando o problema de contaminação ambiental e elevando o risco de exposição de humanos e animais. Alguns estudos relatam que o aumento no consumo 
de agrotóxicos em certas culturas fez com que ocorresse também o aumento no número de pragas, com o surgimento de outras espécies igualmente danosas à cultura (ALVES FILHO, 2002). Isso ocorre porque a introdução de agrotóxicos no ambiente pode provocar efeitos indesejáveis, como a alteração da dinâmica bioquímica natural pela pressão de seleção exercida sobre organismos, tendo como consequência mudanças no funcionamento do ecossistema afetado (SPADOTTO, 2006).

Além do conhecimento das propriedades toxicológicas dos agrotóxicos e da intensidade de uso, informações sobre as principais propriedades físicas e químicas dessas substâncias também são importantes, considerando entendimento dos possíveis processos de retenção, transformação e transporte no ambiente pelas mesmas (SANCHES et al., 2003).

\section{DisPersão de AGROTÓXICO NO AMBIENTE}

A larga utilização de agrotóxicos no processo de produção agropecuária, entre outras aplicações, tem trazido uma série de transtornos e modificações para o ambiente (PERES e MOREIRA, 2007). O uso indiscriminado de agrotóxicos, em qualquer região do planeta, a princípio, gera efeitos locais e regionais. Entretanto, dependendo dos ecossistemas atingidos, tais efeitos provocam diversos reflexos que podem se alastrar por todo um ecossistema (GRISOLIA, 2005).

Somente uma pequena parcela da quantidade dos agrotóxicos aplicados atinge a peste-alvo, sendo que a maior parte tem potencial para se mover para outros compartimentos ambientais, como solo, água, ar e biota (VAN DEN BERG et al., 1999).

Quando aplicados diretamente no solo, os agrotóxicos podem ser degradados por vias químicas, biológica e/ou fotólise. Porém, as moléculas com alta persistência podem permanecer no ambiente sem sofrer nenhuma alteração. Essas moléculas podem ser sorvidas e/ou dessorvidas pelas partículas do solo, sofrer lixiviação e atingir os lençóis subterrâneos ou, ainda, serem levadas para águas superficiais pelo processo de escoamento superficial. Os agrotóxicos presentes na atmosfera, devido a perdas durante a aplicação e volatilização de solo, plantas e águas superficiais, podem retornar aos solos e recursos hídricos superficiais pela deposição seca e/ou úmida (SANCHES et al., 2003). Assim, os recursos hídricos são apontados como um dos compartimentos ambientais mais afetados pelo uso intenso e indiscriminado dos agrotóxicos.

Nos ambientes aquáticos, os pesticidas também podem sofrer sorção ou dessorção das partículas de sedimentos, ser degradados por via química, biológica ou fotólise, além de volatilizarem (SANCHES et al., 2003). Moreira et al. (2002). Em estudo realizado na área rural de Nova Friburgo-RJ, observaram que a dispersão de agrotóxicos no ambiente, em particular nos recursos hídricos, ocasionou impacto significativo na ocorrência e distribuição de espécies animais. Nesse estudo, os organismos associados ao compartimento da coluna d'água foram os mais diretamente afetados.

$\mathrm{Na}$ atmosfera, as moléculas podem ser transportadas por longas distâncias, atingindo áreas distantes dos locais de aplicação (SANCHES et al., 2003). Alguns agrotóxicos clorados, classificados como poluentes orgânicos persistentes, já foram encontrados em áreas remotas como Ártico, Antártida e Monte Everest (MONTONE et al., 2001; BRAUNE et al., 2001; LI et al., 2006; CIPRO, 2007; SILVA, 2009). A dispersão de agrotóxicos de uso atual na atmosfera foi descrita para compostos encontrados em amostras de ar e de água de chuva coletadas em locais distantes dos pontos de aplicação, como área urbana e lagos de altas montanhas (LAABS et al., 2002; SCHEYER et al., 2007; SANTOS et al., 2011).

\section{Agrotóxicos em águas SUPERFICIAIS E SUbTERRÂNEaS}

O nível de poluição e contaminação dos recursos hídricos tem se mostrado crescente (LAABS et al., 2002; SCORZA JÚNIOR et al., 2004; BROWN e VAN BEINUM, 2009). Adisponibilidade de água doce apropriada para consumo humano sem prévio tratamento é cada vez menor e se limita a cada vez mais a fontes de águas minerais. Antes de estar disponível para consumo nas torneiras, a água precisa passar por um rigoroso tratamento e se tornar potável. 
Segundo a Portaria 518/2004 do Ministério da Saúde, água potável é a água para consumo humano cujos parâmetros microbiológicos, físicos, químicos e radioativos atendam ao padrão de potabilidade e que não ofereça riscos à saúde (BRASIL, 2005). Essa mesma portaria regulamenta 54 substâncias químicas que representam riscos à saúde humana, dentre as quais 22 são agrotóxicos (Tabela 1).

Tabela 1 - Padrão de potabilidade para agrotóxicos em água de consumo humano.

\begin{tabular}{lclc}
\multicolumn{1}{c}{ Substância } & $\mathrm{VMP}^{1}\left(\mu \mathrm{g} \mathrm{L}^{-1}\right)$ & \multicolumn{1}{c}{ Substância } & $\mathrm{VMP}^{1}\left(\mu \mathrm{g} \mathrm{L}^{-1}\right)$ \\
\hline Alaclor & 20,0 & Hexaclorobenzeno & 1 \\
Aldrin e Dieldrin & 0,03 & Lindano (g-BHC) & 2 \\
Atrazina & 2 & Metolacloro & 10 \\
Bentazona & 300 & Metoxicloro & 20 \\
Clordano (isômeros) & 0,2 & Molinato & 6 \\
2,4 D & 30 & Pendimetalina & 20 \\
DDT (isômeros) & 2 & Pentaclorofenol & 9 \\
Endossulfam & 20 & Permetrina & 20 \\
Endrim & 0,6 & Propanil & 20 \\
Glifosato & 500 & Simazina & 2 \\
Heptacloro e Heptacloro epóxido & 0,03 & Trifluralina & 20 \\
\hline
\end{tabular}

${ }^{1}$ Valor Máximo Permitido

Fonte: Brasil (2005).

Alguns autores, como Gomes et al., (2001) e Sarcinelli et al. (2005), concordam que a legislação brasileira contempla um número pequeno de agrotóxicos frente à quantidade de princípios ativos utilizados no país. Segundo eles, falta à Portaria 518/ 2004 contemplar alguns princípios ativos de relevância, como os organofosforados e carbamatos, pois estes são largamente utilizados na agricultura brasileira e apresentam alta toxicidade.

O uso de agrotóxicos é um dos mais graves fatores de deterioração da qualidade dos recursos hídricos (AZEVEDO e MONTEIRO, 2011). Estudos em várias regiões do país demonstram que a contaminação dos recursos hídricos por agrotóxicos representa um problema real que pode trazer sérias consequências para o meio ambiente e para a saúde humana (DORES e DE-LAMONICA-FREIRE, 2001; VEIGA et al., 2005).

A Tabela 2 apresenta alguns estudos realizados no Brasil no que se refere à contaminação da água superficial e subterrânea por agrotóxicos. Diferentes classes de agrotóxicos são contempladas; entretanto, o número de analitos determinados ainda é limitado quando se considera o total de princípios ativos com registro para uso no Brasil e a intensidade de uso dos mesmos. 
Tabela 2 - Agrotóxicos em amostras de água superficial e subterrânea no Brasil.

Substâncias detectadas $\quad$ Sup $^{*}$ Sub $^{* *}$ Região de estudo Ref

metomil, maneb, triadimefom, atrazina,

imazapir, etil, metribuzina, simazina,

clo rimurom, fomesafem, glifosato,

X X Primavera do Leste (MT)

flumetsulam, imazetapir e metolacloro.

Tebutiurom

$\alpha-, \beta$ - e sulfato de endossulfam, g lifosato,

tetradifo na, 2,4-D e triclorfom.

benzimidazóis, carbamatos, piretroides e

compostos clorados

$\alpha-, \beta$ - e sulfato de endossulfam, ametrina,

metalacloro, alacloro, trifluralina,

monocrotofós e profenofós

$\begin{array}{lll} & \text { X } & \text { Ribeirão Preto (SP) } \\ \text { X } & \text { X } & \text { Nordeste } \\ \text { X } & \text { X } & \begin{array}{l}\text { Petrolina (PE) e Juazeiro } \\ (\text { BA) }\end{array}\end{array}$

X X Pantanal (MT)

orga no clorado $s$

orga no clorados, organofosforados e piretroides

$\mathrm{X}$

$\mathrm{X}$

diazinona, parationa, pirimifós, malationa,

etiona

organo fosforados e c arbamatos

carbo fur ano e carbaril, simazina, a trazina e

trifluralina

imidaclo prido, a trazina, clomazona

atrazina, simazina, metolacloro, DIA, DEA,

trifluralina

organoclorados

clomazo na, propanil e quincloraque

carbofur ano, quincloraque, clomazona, fipronil

e betaciflutrina

aceta miprido, aldic arbe, c arbenda zim,

carbofur ano, diurom, imidacloprido, metomil e

teflubenzurom

organo clorado s e organo fo sforados

clomazo na, quincloraque, penoxsulam,

imazetapir, imazapir, carbofurano, 3-hydroxy-

carbofur ano, fipronil e tebuconazo 1

atrazina, metolacloro, clorpirifós, $\beta$ e sulfato de

endossulfam, flutriafol e permetrina

(1) D ORES e FREIRE (2001); (2) GOMES et al., (2001); (3) BRITO et al., (2001); (4) FERRACINI et al., (2001); (5) LAABS et al., (2001); (6) RISSATO et al., (2004); (7) SARCINELLI et al., (2005); (8) SANTOS NETO e SIQUEIRA (2005); (9) VEIGA et al., (2005); (10) MARQUES (2005); (11) BORT OLUZZI et al., (2006); (12) DORES et al., (2006); (13) CORBI et al., (2006); (14) MARCHESAN et al., (2007); (15) GRÜTZMACHER et al., (2008); (16) CARBO et al., (2008); (17) MENEZES et al., (2009); (18) SILVA et al., (2009); (19) NOGUEIRA (2011)

* Superficial; ** Subterrânea.

Fonte: Adaptado de Fernandes Neto \& Sarcinelli (2009). 
Esses dados alertam para a contaminação dos recursos hídricos por agrotóxicos. Apesar de muitas vezes o valor da concentração encontrada ser baixo, vários desses agrotóxicos têm a capacidade de bioacumulação, ou seja, possuem afinidade com tecidos lipofílicos e por isso se acumulam no organismo.

A presença de agrotóxicos nos mananciais pode trazer dificuldades para o tratamento daágua em virtude da eventual necessidade de tecnologias mais complexas do que aquelas nor-malmente usadas para a potabilização (MARQUES, 1996; FERNANDES NETO e SARCINELLI, 2009).

\section{Programa de Avaliação de Resíduos de Agrotóxicos em Alimentos - Para}

A ingestão de alimento contendo resíduos de agrotóxicos é uma das principais rotas de exposição humana (MELLO, 1999). Com o objetivo de avaliar os níveis de resíduos de agrotóxicos nos alimentos in natura que chegam à mesa dos consumidores, a
Agência Nacional de Vigilância Sanitária - Anvisa implementou em 2001 o Programa de Análise de Resíduos de Agrotóxicos em Alimentos - Para. As análises do Para têm como parâmetro o Limite Máximo de Resíduos (LMR), que é a quantidade de resíduo legalmente aceita no alimento, conforme aplicação correta do produto no campo (ANVISA, 2011).

Em 2009 o Para apresentou resultados para 3.130 amostras de 20 culturas: abacaxi, alface, arroz, banana, batata, cebola, cenoura, feijão, laranja, maçã, mamão, manga, morango, pimentão, repolho, tomate, uva, couve, beterraba e pepino. Essas culturas foram escolhidas pelos dados de consumo humano obtidos pelo Instituto Brasileiro de Geografia e Estatística - IBGE, pela disponibilidade desses alimentos nos supermercados das diferentes Unidades da Federação e pelo uso intensivo de agrotóxicos nessas culturas. Dependendo da cultura analisada, foram investigados até 234 tipos diferentes de agrotóxicos. A Tabela 3 apresenta os resultados insatisfatórios do Para desde 2002.

Tabela 3 - Resultados insatisfatórios (\%) por culturas do Para, no período de 2002 a 2009.

\begin{tabular}{lcccccccc} 
Cultura\Ano & $\mathbf{2 0 0 2}$ & $\mathbf{2 0 0 3}$ & $\mathbf{2 0 0 4}$ & $\mathbf{2 0 0 5}$ & $\mathbf{2 0 0 6}$ & $\mathbf{2 0 0 7}$ & $\mathbf{2 0 0 8}$ & $\mathbf{2 0 0 9}$ \\
\hline Abacaxi & $*$ & $*$ & $*$ & $*$ & $*$ & $*$ & 9,47 & 44,10 \\
Alface & 8,64 & 6,67 & 14,00 & 46,45 & 28,68 & 40,00 & 19,80 & 38,40 \\
Arroz & $*$ & $*$ & $*$ & $*$ & $*$ & $*$ & 4,41 & 27,20 \\
Banana & 6,53 & 2,22 & 3,59 & 3,65 & $\mathrm{~N}$ & 4,32 & 1,03 & 3,50 \\
Batata & 22,20 & 8,65 & 1,79 & 0 & 0 & 1,36 & 2,00 & 1,20 \\
Cebola & $*$ & $*$ & $*$ & $*$ & $*$ & $*$ & 2,91 & 16,30 \\
Cenoura & 0 & 0 & 19,54 & 11,30 & $\mathrm{~N}$ & 9,93 & 30,39 & 24,80 \\
Feijão & $*$ & $*$ & $*$ & $*$ & $*$ & $*$ & 2,92 & 3,00 \\
Laranja & 1,41 & 0 & 4,91 & 4,70 & 0 & 6,04 & 14,85 & 10,30 \\
Maçã & 4,04 & 3,67 & 4,96 & 3,07 & 5,33 & 2,90 & 3,92 & 5,30 \\
Mamão & 19,50 & 37,56 & 2,50 & 0 & $\mathrm{~N}$ & 17,21 & 17,31 & 38,80 \\
Manga & $*$ & $*$ & $*$ & $*$ & $*$ & $*$ & 0,99 & 8,10 \\
Morango & 46,03 & 54,55 & 39,07 & $\mathrm{~N}$ & 37,68 & 43,62 & 36,05 & 50,80 \\
Pimentão & $*$ & $*$ & $*$ & $*$ & $*$ & $*$ & 64,36 & 80,00 \\
Repolho & $*$ & $*$ & $*$ & $*$ & $*$ & $*$ & 8,82 & 20,50 \\
Tomate & 26,10 & 0 & 7,36 & 4,38 & 2,01 & 44,72 & 18,27 & 32,60 \\
Uva & $*$ & $*$ & $*$ & $*$ & $*$ & $*$ & 32,67 & 56,40 \\
Couve & $*$ & $*$ & $*$ & $*$ & $*$ & $*$ & $*$ & 44,20 \\
Beterraba & $*$ & $*$ & $*$ & $*$ & $*$ & $*$ & $*$ & 32,00 \\
Pepino & $*$ & $*$ & $*$ & $*$ & $*$ & $*$ & $*$ & 54,80 \\
\hline
\end{tabular}

Fonte: Anvisa (2009); Anvisa (2010). 
Observa-se nesses dados a evolução do programa referente ao número de culturas analisadas. $\mathrm{O}$ programa também evoluiu em relação ao número de Estados contemplados com as análises. Em 2002, o Para foi realizado com amostras provenientes de apenas quatro Estados da Federação: Minas Gerais, Paraná, Pernambuco e São Paulo. Já em 2009, 25 Estados e o Distrito Federal foram contemplados. Participaram do Para em 2011 os Estados do Acre, Amapá, Amazonas, Bahia, Ceará, Distrito Federal, Espírito Santo, Goiás, Maranhão, Mato Grosso, Mato Grosso do Sul, Minas Gerais, Pará, Paraíba, Paraná, Pernambuco, Piauí, Rio de Janeiro, Rio Grande do Norte, Rio Grande do Sul, Rondônia, Roraima, Santa Catarina, Sergipe e Tocantins (ANVISA, 2011).

As principais irregularidades encontradas nas amostragens foram: a utilização de agrotóxicos não autorizados (NA) para a cultura $(n=744)$, representando $23,8 \%$ do total; a presença de agrotóxicos em níveis acima do Limite Máximo de Resíduos (LMR) $(\mathrm{n}=88)$, representando 2,8\% do total; e resíduos acima do LMR e NA na mesma amostra $(n=75)$, representando $2,4 \%$ do total (ANVISA, 2011).

As amostras insatisfatórias com níveis de agrotóxicos acima do LMR evidenciam a utilização dos produtos formulados em desacordo com as informações presentes no rótulo e bula. Quanto aos resultados insatisfatórios devido à utilização de agrotóxicos não autorizados, é importante ressaltar que existem dois tipos de irregularidades: amostra na qual foi aplicado um agrotóxico não autorizado para a cultura, mas com o ingrediente ativo com uso permitido em outras culturas, e amostra na qual foi aplicado um agrotóxico de uso proibido no Brasil ou que nunca teve registro no país, ou seja, o seu uso não é permitido em nenhuma cultura. Em relação ao último tipo de irregularidade, 819 amostras apresentaramingredientes ativos não autorizados, dentre as quais, 206 amostras apresentaram ingredientes ativos que se encontram em processo de reavaliação toxicológica no Brasil (ANVISA, 2011).

O mais grave é que, segundo a Anvisa (2011), nos resultados obtidos pelas amostras analisadas pelo Para, observou-se que os agrotóxicos com ingredientes ativos que se encontram em reavaliação vêm sendo utilizados de maneira indiscriminada, sem levar em consideração a existência ou não de registro para determinada cultura. Isto implica duas consequências negativas: a exposição do trabalhador rural aos agrotóxicos que apresentam elevada toxicidade aguda e/ou crônica, motivo pelo qual se encontram em processo de reavaliação pela Anvisa, e o aumento do risco dietético de consumo de resíduos desses agrotóxicos, uma vez que esse uso não foi considerado no cálculo do impacto na Ingestão Diária Aceitável (IDA). Esse risco se agrava à medida que esse agrotóxico é encontrado em um número maior de alimentos comercializados para a população.

A Anvisa divulga anualmente os resultados das análises realizadas pelo Para em seu site com consulta aberta ao público. A população em geral tem conhecimento desses dados também pela sua divulgação nos meios de comunicação. Tendo esse conhecimento, os consumidores deveriam optar em consumir alimentos que tenham a origem identificada, pois isso aumenta o comprometimento dos produtores em relação à qualidade dos alimentos produzidos atendendo às recomendações das Boas Práticas Agrícolas. Além disso, deve-se optar por consumir alimentos da época ou produzidos por métodos de produção integrada, que a princípio recebem uma carga menor de agrotóxicos. Consumir alimentos orgânicos também pode reduzir a exposição dietética aos agrotóxicos, além de contribuir para a manutenção de uma cadeia de produção de alimentos ambientalmente mais saudável (ANVISA, 2011).

De acordo com a Anvisa (2011), algumas ações foram iniciadas no sentido de promover a capacitação de diferentes segmentos da sociedade, como a criação do Grupo de Trabalho de Educação e Saúde sobre Agrotóxicos (Gesa). Integrado por diferentes órgãos e entidades, seu objetivo é elaborar propostas e ações educativas para reduzir os impactos do uso de agrotóxicos na saúde da população, implementar ações e estratégias para incentivar os sistemas orgânicos de 
produção ou outros sistemas alternativos para o uso de agrotóxicos e, no caso dos cultivos convencionais, orientar o uso racional de agrotóxicos.

\section{ACUmulação no ORganismo humano}

A saúde humana pode ser afetada pelos agrotóxicos pelo contato direto com essas substâncias. Não somente os moradores do meio rural estão expostos aos agrotóxicos; os do meio urbano também se encontram sob risco, devido à contaminação ambiental e dos alimentos. Aavaliação da contaminação ambiental é de fundamental importância para a compreensão da contaminação humana por agrotóxicos (MOREIRA et $a l .$, 2002), uma vez que os seres humanos podem receber resíduos provenientes de matrizes ambientais contaminadas, como água e ar. Além disso, o homem se encontra no final da cadeia alimentar, o que faz com que ele receba uma quantidade maior de substâncias químicas, inclusive os agrotóxicos estáveis que se foram acumulando ao longo de toda a cadeia.

Vários estudos evidenciam a exposição e contaminação humana por agrotóxicos. Koifman et al. (2002) verificaram correlação positiva entre a exposição da população aos agrotóxicos consumidos na década de 1980 e os distúrbios reprodutivos observados na década de 1990. O estudo realizado em 11 Estados brasileiros observou coeficientes de correlação moderado e alto entre venda de agrotóxicos e mortalidade por câncer de mama, principalmente entre as mulheres de 50-69 anos de idade, entre 1995 e 1997, e mortalidade por câncer de ovário.

Alguns estudos toxicológicos com animais apresentam evidências que altas doses de alguns agrotóxicos podem alterar a função reprodutiva e produzir malformações congênitas (HEEREN et al., 2003; CALVERT et al., 2007). Na África do Sul, mulheres expostas aos agrotóxicos apresentaram um risco sete vezes maior de ter filhos com alguma malformação congênita do que mulheres não expostas a essas substâncias, sugerindo uma relação entre a exposição aos agrotóxicos e o nascimento de crianças com malformações congênitas (HEEREN et al., 2003).
Foi verificada na Espanha a existência de associação entre a exposição paterna a agrotóxicos durante o primeiro trimestre da gravidez e o risco de morte fetal a partir de malformações congênitas (REGIDOR et al., 2003). Cooper et al. (2006) verificaram que mães expostas a inibidores da acetilcolinesterase no primeiro trimestre de gravidez tinham um risco 2,7 vezes maior de ter filhos com alguma malformação congênita do que mães não expostas a essas substâncias.

Sergeev e Carpenter (2005) avaliaram as taxas de hospitalização por doenças coronarianas e infarto agudo do miocárdio e a proximidade da residência de áreas contaminadas por poluentes orgânicos persistentes e outros poluentes no Estado de Nova York, nos Estados Unidos.

Um estudo feito com moradores da zona urbana e rural de Lucas do Rio Verde/MT mostrou a presença dos agrotóxicos em sangue e urina de moradores das duas populações. Os pesquisadores encontraram glifosato, muito utilizado na região, piretroides e alguns compostos organoclorados ( $p, p^{\prime}-$ DDE, o,p'- DDT, p,p'- DDT, $\beta$-HCH e aldrim) na urina de moradores dos dois grupos pesquisados. Os níveis de glifosato encontrados na população rural foram duas vezes superiores aos níveis da população urbana. O contrário foi observado para os níveis de piretroides, indicando uma possível influência dos agrotóxicos domissanitários. Como para o glifosato, os níveis de organoclorados também foram maiores na população rural do que na população urbana (MOREIRA et al, 2010).

Agrotóxicos de diferentes classes químicas, principalmente organoclorados, também têm sido encontrados em leite materno de mães residentes em diferentes regiões do Brasil, como Ribeirão Preto/SP (MATUO et al., 1992), Cuiabá/MT (OLIVEIRA, 1997), Duque de Caxias/RJ (MELLO, 1999), Rio de Janeiro/RJ (PAUMGARTTEN et al., 2000), Rio de Janeiro e Grande Rio/RJ (MESQUITA, 2001), São Paulo/SP e Belo Horizonte/MG (KRAUSS et al., 2004), Porto alegre/RS (CALEFFI, 2005), Porto Velho e Axinim/RO (AZEREDO et al., 2008) e Lucas do Rio Verde/MT (PALMA, 2011). Esses estudos 
evidenciam a contaminação do leite materno, que, embora considerado como veículo de eliminação desses compostos do organismo, é um importante alimento para os recém-nascidos.

\section{EXPOSIÇÃO E INTOXICAÇÃo HUMANA POR AGROTóxICOS}

A influência do ambiente sobre a saúde humana é inquestionável. Essas relações incorporam todos os elementos e fatores que potencialmente afetam a saúde, incluindo, entre outros, a exposição a agentes específicos como substâncias químicas e elementos biológicos (TAMBELLINI e CÂMARA, 1998; PORTO, 2007).

A população em geral pode estar exposta aos agrotóxicos pela ingestão de alimentos e água contaminada e pelas exposições inalatória e dérmica, após aplicações domésticas ou pulverizações em culturas ou ambientes públicos (NUNES e TAJARA, 1998).

Estudos têm relatado associações estatísticas positivas entre a exposição humana a agrotóxicos e problemas de saúde, tais como aumento de certos tipos de câncer de mama e/ou do trato reprodutivo, redução da fertilidade masculina, anormalidades no desenvolvimento sexual, malformações entre outros (MEYER et al., 1999; KOIFMAN et al., 2002; HEEREN et al., 2003; REGIDOR et al., 2003; SERGEEV e CARPENTER 2005; COOPER et al., 2006; CALVERT et al., 2007).

Alguns agrotóxicos atuam como interferentes endócrinos. Segundo a União Europeia, os interferentes endócrinos podem danificar diretamente um órgão endócrino ou alterar a sua função, interagir com um receptor de hormônios ou alterar o metabolismo de um hormônio em umórgão endócrino (ECE, 2010). As substâncias sintéticas que apresentam essa ação são geralmente persistentes no ambiente, podendo acumular-se ao longo da cadeia trófica.

Além dos efeitos crônicos, ou seja, que surgem depois de um longo período de exposição, existem também os efeitos agudos, que são consequência de uma exposição única ou no curto prazo, e resultam usualmente em dados clínicos evidentes. Essas ocorrências podem ser acidentais, como também intencionais (ROMÃO e VIEIRA, 2004).

Os sinais de intoxicação aguda, devido à exposição aos agrotóxicos, incluem transtornos de visão, vômitos, ansiedade, confusão mental, hipertensão arterial, efeitos neurológicos diversos e até mesmo a morte.

No Brasil, os dados sobre intoxicação humana pelos agrotóxicos são disponibilizados pelo Sistema Nacional de Informações Tóxico-Farmacológicas Sinitox, criado em 1980 e vinculado à Fiocruz, que coordena a coleta, a análise e a divulgação de dados sobre envenenamentos ocorridos no Brasil anualmente. O agrotóxico é o terceiro principal agente tóxico em relação ao número de casos de intoxicação humana registrados pelo Sinitox (BOCHNER, 2007). A Figura 1 apresenta os dados de intoxicação por agrotóxicos agrícola e domiciliar no Brasil no período entre 1999 e 2009. 


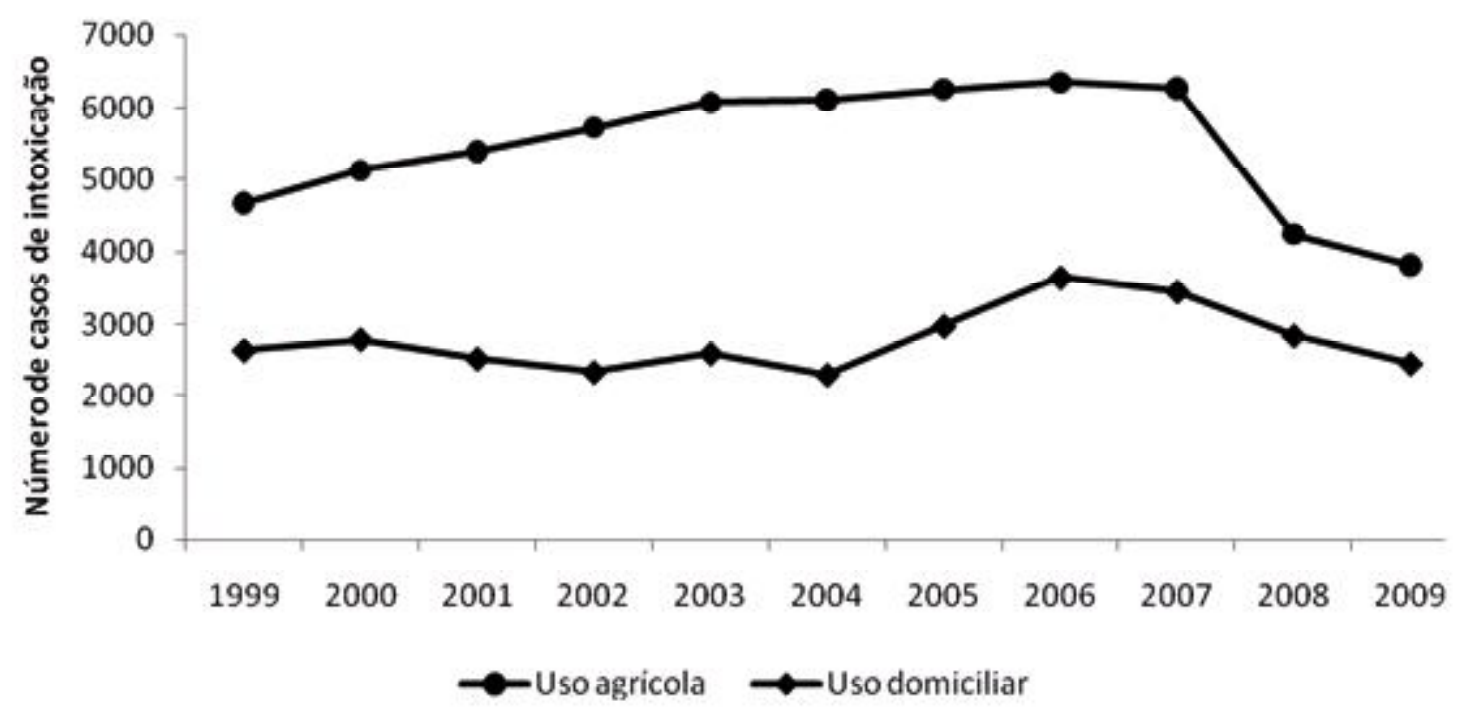

Figura 1 - Intoxicação por agrotóxicos agrícola e domiciliar no Brasil entre 1999 e 2009.

Fonte: Sinitox (2011)

É importante ressaltar que os dados apresentados no Sinitox não representam a totalidade de casos ocorridos no Brasil, uma vez que a notificação dos casos de intoxicação por agrotóxicos realizada pelos Centros de Informação e Assistência Toxicológica Ciats ao Sinitox é voluntária, o que gera irregularidades nas estatísticas divulgadas por esse sistema (BOCHNER, 2007).

É perceptível uma queda no número de casos de intoxicação a partir de 2007. Pela inconsistência dos dados, mencionada anteriormente, é difícil relatar a verdadeira causa dessa diminuição. Duas hipóteses podem ser levantadas: o número de subnotificações pode ter aumentado a partir desse período e/ou os cursos sobre o "uso seguro do agrotóxico" realizados pelas revendedoras de agrotóxicos aos produtores rurais têm surtido efeito.

\section{Considerações finais}

$\mathrm{Na}$ busca por grandes produções, o processo produtivo agrícola adotado no Brasil, com intensa aplicação de agrotóxicos, leva o país a um desenvolvimento insustentável, uma vez que degrada o ambiente, poluindo os recursos hídricos, o solo, o ar e, consequentemente, expondo a população aos agrotóxicos.

Considerando os diversos problemas de saúde que podem ocorrer devido à exposição constante aos agrotóxicos e o direito previsto na "Constituição Federal" (Art. $225 \mathrm{CF}$ ) de viver em um ambiente ecologicamente equilibrado, é papel da população estar ciente de tais problemáticas e cobrar das autoridades maior fiscalização no uso desses compostos.

Os agrotóxicos podem se acumular no organismo e causar diversos danos à saúde, sendo que muitos deles ainda não são totalmente conhecidos. Assim, a problemática do uso intensivo de agrotóxico deve ser debatida entre União e sociedade para que medidas urgentes sejam tomadas e danos no futuro sejam evitados.

\section{REFERÊNCIAS}

ALVES FILHO, J.P. Uso de agrotóxicos no

Brasil: controle social e interesse corporativo. São Paulo: Annablume, 2002. 188p.

ANVISA - Agência Nacional de Vigilância Sanitária. Monografia do ingrediente ativo deltametrina. Brasília (DF); 2011. Disponível em: 
Agrotóxicos em água e alimentos...

<http://portal.anvisa.gov.br/wps/portal/anvisa/home/ agrotoxicotoxicologia?cat=Programa+de+ Analise+de+Residuos+de+Agrotoxicos+ em+Alimentos\&cat 1>. Acesso em: 14 maio. 2011.

AZEREDO, A; TORRES, J.P.M; FONSECA, M.F; BRITTO, J.L; BASTOS, W.R; AZEVEDO E SILVA, C.E, et al. DDT and its metabolites in breast milk from the Madeira River basin in the Amazon, Brazil. Chemosphere, v. 73, p. 246-251. 2008.

AZEVEDO, A.A.; MONTEIRO, J.L.G. Análise dos impactos ambientais da atividade agropecuária no cerrado e suas inter-relações com os recursos hídricos na região do pantanal - World Wildlife Fund. Disponível em: <http://ssets.wwfbr.panda.org/ downloadswwf_brasil_impactos_atividade_ agropecuaria_cerrado_pantanal.pdf $>$. Acesso em: 21 mai. 2011.

BOCHNER, R. Sistema Nacional de Informações Tóxico-Farmacológicas - Sinitox e as intoxicações humanas por agrotóxicos no Brasil. Ciência e Saúde Coletiva, v. 12, n. 1, p. 73-89. 2007.

BORTOLUZZI, E.C; RHEINHEIMER, D.S; GONÇALVES, C.S, et al. Contaminação de águas superficiais por agrotóxicos em função do uso do solo numa microbacia hidrográfica de Agudo, RS. Revista Brasileira de Engenharia Agrícola e Ambiental, v. 10, n. 4, p. 881-887, 2006.

BRASIL. Ministério da Saúde. Portaria MS n. ${ }^{\circ}$ 518, de 25 de março de 2004. Estabelece os procedimentos e responsabilidades relativos ao controle e vigilância da qualidade da água para consumo humano e seu padrão de potabilidade, e dá outras providências. Brasília, DF: Ministério da Saúde, 2005.

BRAUNE, B.M; DONALDSON, G.M; HOBSON, K.A. Contaminant residues in seabird eggs from the
Canadian Artic. Part 1. Temporal trends 1975-1998. Environmental Pollution, v. 114, p. 39-54, 2001.

BRITO, N.M; AMARANTE JR, O.P; ABARKELI, R; SANTOS, T.C.R; RIBEIRO, M.L. Risco de contaminação de águas por pesticidas aplicados em plantações de eucaliptos e coqueiros: análise preliminar. Pesticidas: Revista de Ecotoxicologia e Meio Ambiente, v. 11, p. 93-104, 2001.

BROWN, C. D.; VAN BEINUM, W. Pesticide transport via sub-surface drains in Europe.

Environmental Pollution, v. 157, n. 12, p. 33143324, 2009.

CALDAS, E.D.; SOUZA, L. C. de. Avaliação de risco crônico da ingestão de resíduos de pesticidas na dieta brasileira. Revista de Saúde Pública, v. 34, n. 5, p. 529-537, 2000.

\section{CALEFFI, G.H. Resíduos organoclorados em} sangue, leite materno e tecido adipose humanos em regiões definidas do estado do Rio Grande do Sul, Brasil. 2005. 149 f. Dissertaçao (Mestrado em Ecologia) - Universidade Federal do Rio Grande do Sul, Porto Alegre, 2005.

CALVERT, G.M; ALARCON, W.A; CHEMINSKI, A; CROWLEY, M.S; BARRETT, R; CORREA, A, et al. Case Report: Three Farmworkers Who Gave Birth to Infants with Birth Defects Closely Grouped in Time and Place-Florida and North Carolina, 2004-2005. Environ Health Perspectives, v. 115, p. 787-791, 2007.

CARBO, L; SOUZA, V; DORES, E.F.G.C; RIBEIRO, M.L. Determination of Pesticides Multiresidues in Shallow Groundwater in a Cottongrowing Region of Mato Grosso, Brazil. Journal of the Brazilian Chemical Society, v. 19, n. 6, p. 1111-1117, 2008.

CIPRO, C.V.Z. Ocorrência de compostos 
organoclorados em Euphausia superba e em ovos gorados de pinguins do gênero Pygoscelis. 2007. 133 f. Dissertação (Mestrado em Oceanografia Química e Geológica) - Universidade de São Paulo, São Paulo, 2007.

COOPER, W.O; HERNANDEZ-DIAZ, S; ARBOGAST, P.G; DUDLEY, J.A; DYER, S; GIDEON, O.S, et al. Major congenital malformations after first-trimester exposure to ACE inhibitors. New England Journal of Medicine, v. 354, n. 23, p. 2443-2451, 2006.

CORBI, J.J. et al. Diagnóstico ambiental de metais e organolclorados em córregos adjacentes a áreas de cultivo de cana-de-açúcar (Estado de São Paulo, Brasil). Química Nova, v. 29, p.61-65, 2006.

DORES, E.F.G.C; DE-LAMONICA-FREIRE, E.M. Contaminação do ambiente aquático por pesticidas. Estudo de caso: águas usadas para consumo humano em Primavera do Leste, Mato Grosso - análise preliminar. Química Nova, v. 24, n. 1, p. 27-36, 2001.

DORES, E.F.G.C; NAVICKIENE, S; CUNHA, M.L.F; CARBO, L. RIBEIRO, M.L; DELAMONICA-FREIRE, E.M. Multiresidue Determination of Herbicides in Environmental Waters from Primavera do Leste Region (Middle West of Brazil) by SPE-GC-NPD. Journal of the Brazilian Chemical Society, v. 17, n. 5, p. 866-873, 2006.

ECE - European Commission Environment.

Endocrine Disrupters Website Disponível em: <http:/ /ec.europa.eu/environment/endocrine/documents/ reports_en.htm>.Acesso em: 17 nov. 2010.

FERNANDES NETO, M.L; SARCINELLI, P.N. Água para consumo humano: uma abordagem de avaliação de risco e contribuição ao processo de atualização da legislação brasileira. Engenharia Sanitária e Ambiental, v. 14, p.69-78, 2009.
FERRACINI, V.L.; PESSOA, M.C.Y.P.; SILVA, A.S.; SPADOTTO, C.A. Análise de risco de contaminação das águas subterrâneas e superficiais da região de Petrolina (PE) e Juazeiro (BA). Pesticidas: Revista de Ecotoxicologia e Meio Ambiente, v. 11, p.1-16, 2001.

GOMES, M.A.F; SPADOTTO, C.A; LANCHOTTE, V.L. Ocorrência do herbicida Tebuthiuron na água subterrânea da microbacia do Córrego Espraiado, Ribeirão Preto, SP. Pesticidas: Revista de Ecotoxicologia e Meio Ambiente, v. 11, p. 65-76, 2001.

GRISOLIA, C.K. Agrotóxicos: Mutações, Câncer e Reprodução. Brasília: Unb, 2005. 392p.

GRUTZMACHER, D.D; GRUTZMACHER, A.D; AGOSTINETTO, D; LOECK, A.C; ROMAN, R, et al. Monitoramento de agrotóxicos em dois mananciais hídricos no sul do Brasil. Revista Brasileira de Engenharia Agrícola e Ambiental, v. 12, p. 632-637, 2008.

GUNNINGHAM, N.; SINCLAIR, D. Policy instrument choice and diffuse source pollution. Journal Environmental Law, v. 17, p.51-81, 2005.

HEEREN, G.A; TYLER, J; MANDEYA, A. Agricultural chemical exposures and birth defects in the Eastern Cape Province, South Africa A case control study. Environmental Health: A Global Access Science Source. v. 2, 2003.

KOIFMAN, S; KOIFMAN, R.J; MEYER, A. Human reproductive system disturbances and pesticide exposure in Brazil. Cadernos de Saúde Pública, v. 18, n. 2, p. 435-445, 2002.

KRAUSS, T; BRAGA, A.M.C.B; ROSA, J.M; KYPKE, K; MALISCH, R. Levels of organochlorine in Brazilian human Milk. Organohalogen Compounds, v. 66, p. 2773-2777, 2004. 
Agrotóxicos em água e alimentos...

LAABS, V; AMELUNG, W; PINTO, A.A; WANTZEN, M; SILVA, C.J; ZECH, W. Pesticides in Surface Water, Sediment, and Rainfall of the Northeastern Pantanal Basin, Brazil. Journal of Environmental Quality, v.31, p.1636-1648, 2002.

LI, J; ZHU, T; WANG, F; QIU, X.H; LIN, W.L. Observation of organochlorine pesticides in the air of the Mt. Everest region. Ecotoxicology and Environmental Safety, v. 63, p. 33-41, 2006.

MARCHESAN, E; ZANELLA, R; AVILA, L.A; CAMARGO, E.R; MACHADO, S.L.O;

MACEDO, V.R.M. Rice herbicide monitoring in two brazilian rivers during the rice growing season.

Scientia Agricola, v. 64, n. 2, p. 131-137, 2007.

MARQUES, J.F. Pesticidas na água potável: padrões de qualidade e princípio de precaução na legislação europeia. Caderno CRH, v. 24/25, p. 269-285, 1996.

MARQUES, M.N. Avaliação do impacto de agrotóxicos em áreas de proteção ambiental, pertencentes à bacia hidrográfica do Rio Ribeira de Iguape, São Paulo. Uma contribuição à análise crítica da legislação sobre o padrão de potabilidade. 2005. $198 \mathrm{f}$. Tese (Doutorado em Ciências na Área de Tecnologia) - Instituto de Pesquisas Energéticas e Nucleares, São Paulo, 2005.

MATUO, Y.K; LOPES, J.N.C; CASANOVA, I.C; MATUO, T; LOPES, J.L.C. Organochlorine pesticide residues in human milk in the Ribeirão Preto Region, State of São Paulo, Brazil. Archives of Environmental Contamination and Toxicology, v. 22, p. 167-175, 1992.

MELLO, J.L. Avaliação da contaminação por HCH e DDT, dos leites de vaca e humano, provenientes da cidade dos meninos, Duque de Caxias-RJ. 1999. 129 f. Dissertação (Mestrado em Saúde Pública) - Escola Nacional de Saúde Pública da Fundação Oswaldo Cruz, Rio de Janeiro, 1999.

MENEZES, J.M; PRADO, R.B; SILVA Jr, G.C; MANSUR, K.L; OLIVEIRA, E.S. Qualidade da água e sua relação espacial com as fontes de contaminação antrópicas e naturais: bacia hidrográfica do rio São domingos - RJ. Engenharia Agrícola, v. 29, n. 4, p. 687-698, 2009.

MESQUITA, A.S. Avaliação da contaminação do leite materno por agrotóxicos organoclorados persistentes em mulheres doadoras do Banco de leite do Instituto Fernandes Figueira, RJ. 2001. 68 f. Dissertação (Mestrado em Saúde Pública) - Escola Nacional de Saúde Pública da Fundação Oswaldo Cruz, Rio de Janeiro, 2001.

MENTEN, J.O.M; SAMPAIO, I.A; MOREIRA, H; FLÔRES, D; MENTEN, M. O setor de defensivos agrícolas no Brasil. Disponível em: <http://www.sindag.com.br/dados_mercado.php>. Acesso em: 25 mai. 2011.

MEYER, A; SARCINELLI, P.N; MOREIRA, J.C. Estarão alguns grupos populacionais brasileiros sujeitos à ação de disruptores endócrinos? Caderno de Saúde Pública, v. 15, n. 4, p. 845-850, 1999.

MOREIRA, J.C; JACOB, S.C; PERES, F; LIMA, J.S; MEYER, A, OLIVEIRA-SILVA, J.J et al.

Avaliação integrada do impacto do uso de agrotóxicos sobre a saúde humana em uma comunidade agrícola de Nova Friburgo, RJ. Ciência e Saúde Coletiva, v. 7, n. 2, p. 299-311, 2002.

MOREIRA, J.C; PERES, F; PIGNATI, W.A; DORES, E.F.G.C. Relatório de Pesquisa: Avaliação do risco à saúde humana decorrente do uso de agrotóxicos na agricultura e pecuária na Região Centro-Oeste. 2010. Processo CNPq 555193/2006-3. 
MONTONE, R.C; TANIGUCHI, S; WEBER, R.R. Polychlorinated biphenyls in marine sediments of Admiralty Bay, King George Island, Antarctica. Marine Pollution, v. 42, p.611-614, 2001.

NOGUEIRA, E.N. Pesticidas e Recursos Hídricos: validação de método analítico e avaliação da distribuição em dois pólos agrícolas de Mato Grosso, 2011. $112 \mathrm{f}$. Dissertação (Mestrado em Recursos Hídricos) Universidade Federal de Mato Grosso, Cuiabá, 2011.

NUNES, M.V; TAJARA, E.H. Efeitos tardios dos praguicidas organoclorados no homem. Revista de Saúde Pública, v. 32, n. 4, p. 372-383, 1998.

OLIVEIRA, M.A.G. Níveis de resíduos de praguicidas organoclorados no leite de mães de uma população de Cuiabá-MT. 1997. Dissertação (Mestrado em Saúde Coletiva) - Universidade Federal de Mato Grosso, Cuiabá, 1997.

\section{PALMA, D.C.A. Agrotóxicos em leite humano} de mães residentes em Lucas do Rio VerdeMT. 2011. 103 f. Dissertação (Mestrado em Saúde Coletiva) - Universidade Federal de Mato Grosso, Cuiabá, 2011.

PAUMGARTTEN, F.J.R; CRUZ, C.M; CHAHOUD, I; PALAVINSKAS, R; MATHAR, W. PCDDS, PCDFS, PCBS, and other organochlorine compounds in human milk from Rio de Janeiro, Brazil. Environmental Research Section, v. 83, p. 293-297, 2000.

PERES, F; MOREIRA, J.C; CLAUDIO, L. Os impactos dos agrotóxicos sobre a saúde e o ambiente. Ciência e Saúde Coletiva, v. 12, 2007.

PERES, F; MOREIRA, J.C. Saúde e ambiente em sua relação com o consumo de agrotóxicos em um pólo agrícola do Estado do Rio de Janeiro, Brasil.
Caderno de Saúde Pública, v. 23 n. 4, p.612-621, 2007.

PORTO, M.F. Agrotóxicos, Saúde Coletiva e Insustentabilidade: Uma Visão Crítica Da Ecologia Política. Ciência e Saúde Coletiva, v. 12, n. 1, p. 17-20, 2007.

REGIDOR, E; RONDA, E; GARCÍA, A.M; DOMÍNGUEZ, V. Paternal exposure to agricultural pesticides and cause specific fetal death.

Occupational Environmental Medicine, v. 64, p. 334-339, 2004.

RISSATO, S.R.; LIBÂNIO, M.; GIAFFERIS, G.P.; GERENUTTI, M. Determinação de pesticidas organoclorados em água de manancial, água potável e solo na região de Bauru, SP. Química Nova, v. 27, p. 739-743, 2004.

ROMÃO, M. R; VIEIRA, L. J. E. S. Tentativas de suicídio por envenenamento. Revista Brasileira em Promoção da Saúde, v. 17, p. 14-20, 2004.

SANCHES, S.M; SILVA, C.H.T.P; CAMPOS, S.X; VIEIRA, E.M. Pesticidas e seus respectivos riscos associados à contaminação da água.

Pesticidas: Revista de Ecotoxicologia e Meio Ambiente, v. 13, p. 53-58, 2003.

SANTOS L.G, LOURENCETTI C, PINTO A.A, PIGNATI W.A, DORES E.F.G.C. Validation and application of na analytical method for determining pesticides in the gas phase of ambienta air. Journal of Environmental Science and Health. Part B, Pesticides, Food Contaminants, and Agricultural Wastes, v. 46, p. 150-162, 2011.

SANTOS NETO, A.J; SIQUEIRA, M.E.P.B. Análise de praguicidas organofosforados em água por extração em fase sólida (SPE) utilizando discos C18 e cromatografia em fase gasosa: avaliação da contaminação do reservatório de furnas (MG- 
Brasil). Química Nova, v. 28, p. 747-750, 2005. SARCINELLI, P.N. et al. Estudo dos agrotóxicos mais utilizados no país: avaliação da contaminação das águas de consumo humano nas grandes bacias hidrográficas e estratégias analíticas para o monitoramento. Relatório Final. Rio de Janeiro: Fiocruz, 2005.

SCHEYER, A.; MORVILLE, S.; MIRABEL, P.; MILLET, M. Variability of atmospheric pesticide concentrations between urban and rural areas during intensive pesticide application. Atmospheric

Environment, v. 41, p. 3604-3618, 2007.

SCORZA JÚNIOR, R. P.; SMELT, J. H.; BOESTEN, J. J. T. I.; HENDRIKS, R. F. A.; VAN DER ZEE, S. E. A. T. M. Preferential flow of bromide, bentazon, and imidacloprid in a Dutch clay soil. Journal of Environmental Quality, v. 33, n. 4, p. 1473-1486, 2004.

\section{SERGEEV, A.V; CARPENTER, D.O.}

Hospitalization rates for coronary heart disease in relation to residence near areas contaminated with persistent organic pollutants and other pollutants.

Environmental Health Perspectives, v. 113, n. 6, p. 756-761, 2005.

\section{SILVA, G.R. Níveis de Agrotóxicos} Organoclorados e Perfil Alimentar na Cidade dos Meninos Duque de Caxias, RJ, Brasil, entre 2003 e 2004. 2009. 81 f. Dissertação (Mestrado em Ciências na Área de Saúde Pública e
Meio Ambiente) - Fundação Oswaldo Cruz, Rio de Janeiro, 2009.

SILVA, D.R.O; AVILA, L.A; AGOSTINELLO, D; MAGRO, T.D, et al. Monitoramento de agrotóxicos em águas superficiais de regiões orizícolas no sul do Brasil. Ciência Rural, v. 39, p. 2383-2389, 2009.

SPADOTTO, C.A. Abordagem interdisciplinar na avaliação ambiental de agrotóxicos. Revista científica eletrônica NPI. Disponivelem: < http:// www.fmr.edu.br/npi/003.pdf $>$. Acesso em: 26 mai. 2011.

TAMBELLINI, A.T; CÂMARA, V.M. A temática saúde e ambiente no processo de desenvolvimento do campo da saúde coletiva: aspectos históricos, conceituais e metodológicos. Ciência e Saúde Coletiva, v. 3, n. 2, p. 47-59, 1998.

VAN DEN BERG, F.; KUBIAK, R.; BENJEY, W. G.; MAJEWSKI, M. S.; YATES, S. R.; REEVES, G. L.; SMELT, J. H.; VAN DER LINDEN, A. M. A. Emission of pesticides into the air. Water, Air and Soil Pollution, v. 115, n. 1-4, p. 195-218, 1999.

VEIGA, M.M; SILVA, D.M; VEIGA, L.B.E. Análise do Risco de Contaminação Ambiental por Agrotóxicos nos Sistemas Hídricos do Município de Paty do Alferes, RJ. In: ENCONTRO NACIONAL DE ENGENHARIA DE PRODUÇÃO, 25., 2005; Porto Alegre, p. 4259-4264.

RECEBIDO EM 18/8/2011

Aсегто EM 29/11/2011 